\title{
Is the cochlear implant a successful long-term solution for single-sided deaf and asymmetric hearing-impaired patients?
}

\author{
Iva Speck $^{1}$ (D) $\cdot$ Pascal Challier $^{1} \cdot$ Thomas Wesarg $^{1} \cdot$ Till Fabian Jakob $^{1} \cdot$ Antje Aschendorff $^{1} \cdot$ Frederike Hassepass $^{1}$. \\ Susan Arndt ${ }^{1}$
}

Received: 29 June 2020 / Accepted: 28 September 2020 / Published online: 16 October 2020

(c) The Author(s) 2020

\begin{abstract}
Purpose We investigated the long-term results of cochlear implant (CI) recipients with asymmetric hearing loss (AHL) or single-sided deafness (SSD). We focused on wearing behavior, audiometric hearing rehabilitation, and subjective benefits of the CI. CI is expected to improve audiological results, subjective hearing perception, and tinnitus burden.

Methods Speech recognition in background noise and sound localization were assessed preoperatively and after at least six years of CI experience. Validated questionnaires determined the subjective benefit of CI use and the subjective evaluation of tinnitus.

Results Over $80 \%$ of the included AHL and SSD CI recipients used their CI between 6 and 10 h daily; four subjects with SSD were non-users. Speech recognition in background noise and sound localization improved significantly compared with the unaided preoperative situation. Additionally, CI improved subjective speech intelligibility and spatial hearing impression while reducing tinnitus burden.

Conclusion Subjects with AHL and SSD benefit from CI, subjectively and audiologically. Cochlear implant is a successful long-term treatment for AHL and SSD.
\end{abstract}

Keywords Single-sided deafness $\cdot$ Asymmetric hearing loss $\cdot$ Cochlear Implant $\cdot$ Long term

Abbreviations
CI
$\begin{array}{ll}\text { Cochlear implant } \\ \text { NH } & \text { Normal hearing } \\ \text { AHL } & \text { Asymmetric hearing loss } \\ \text { SSD } & \text { Single-sided deafness } \\ \text { PTA4 } & \text { Pure-tone average for the frequencies 500, 1000, } \\ & \text { 2000, and 4000 Hz } \\ \text { OLSA } & \text { Oldenburg sentence test } \\ \text { SSQ } & \text { Speech, Spatial and Qualities of Hearing Scale } \\ \text { NRS } & \text { Numerical rating scale }\end{array}$

Iva Speck

iva.speck@uniklinik-freiburg.de

1 Faculty of Medicine, Department of Otorhinolaryngology-

Head and Neck Surgery, Medical Center-University

of Freiburg, Killianstraße 5, 79106 Freiburg, Germany

\section{Introduction}

The extreme asymmetry of hearing impairs individuals with single-sided deafness (SSD) and asymmetric hearing loss (AHL) in their speech recognition in noise and the localization of sound sources [1-5]. SSD and AHL can also cause psychological health issues, e.g., high-stress levels, high self-reported listening effort, low self-efficiency, and low self-confidence, resulting in exhaustion, frustration, and social withdrawal [5-8]. In addition, many AHL and SSD patients experience disturbing tinnitus [2, 9-11].

Cochlear implants (CIs) improve speech recognition in noise and the localization of sound sources in patients with SSD [12-15]. CIs also reduce the cognitive load and improves the anxiety states and hearing-related quality of life of such patients $[1,2,10,11,16]$.

In the first and, to date, only long-term study on SSD CI recipients, Távora-Vieira and colleagues compared the conditions of CI turned on and CI turned off at the time of long-term measurement (follow-up of 4-10 years) [14].

Our aim was to determine the long-term success of CI in subjects with SSD and AHL by comparing preoperative with 
long-term measurements. To investigate successful treatment, we collected the following data: the wearing behavior of the CI, speech recognition in noise, the localization of sound sources, the subjective evaluation of hearing, and the tinnitus burden. We expected continuous use in cases of treatment success and improvement of the audiological results, subjective hearing and tinnitus burden.

\section{Materials and methods}

The Ethics Committee of the Albert-Ludwigs-University of Freiburg (No. 3/17) approved the present study. We registered the study with the German Register of Clinical Studies (DRKS00017632).

\section{Participants}

We derived the criteria for SSD and AHL from the consensus papers of Vincent et al. [17] and Van de Heyning et al. [18]. These consensus papers require the poorer-hearing ear to have an unaided hearing threshold of $\geq 70 \mathrm{~dB}$ HL up to and including $4 \mathrm{kHz}$. For the better-hearing ear, we applied the definition by Vincent and colleagues [17], namely SSD participants: hearing threshold of $\leq 30 \mathrm{~dB}$ HL in the frequencies up to and including $4 \mathrm{kHz}$; AHL participants: hearing impairment of $>30 \mathrm{~dB} \mathrm{HL}$ and $\leq 60 \mathrm{~dB}$ HL up to and including $4 \mathrm{kHz}$ [17]. The interaural asymmetry was required to be equal to or more than $30 \mathrm{~dB}$ [17].

We included German-speaking adults treated at the ENT University Hospital Freiburg between 2008 and 2013. "Long-term" was defined as a period of five or more years after the initial fitting of the CI and, in the present study, ranged from six to eleven years.

\section{Data collection}

We compared long-term measurements with preoperative measurements. We chose the measurements that Vincent et al. and Van de Heyning et al. proposed as evidence of the therapeutic success of CI in subjects with SSD and AHL $[17,18]$.

\section{Audiometric measurement}

We performed bone- and air-conduction pure-tone audiometry and the unaided Freiburg monosyllable test in $\%$ at $65 \mathrm{~dB}$ SPL. Audiometric data were compared using the pure-tone average for the frequencies 500,1000, 2000, and $4000 \mathrm{~Hz}$ (pure-tone average, PTA4).

Speech recognition in background noise was tested with the Oldenburg sentence test (OLSA) [19, 20]. The 50\% speech intelligibility threshold was determined adaptively with a fixed noise level of $65 \mathrm{~dB}$ SPL and an initial speech level of $65 \mathrm{~dB}$ SPL. We examined three presentation conditions: S0N0, SnhNssd, and SssdNnh, as previously described by Arndt and colleagues [2]. In the presentation condition S0N0, both speech and noise were displayed from the front at an angle of $0^{\circ}$. In the presentation condition SnhNssd, the speech was presented from the better-hearing side and background noise from the poorer-hearing side at an angle of $45^{\circ}$. Speech and noise presentation was vice versa in the presentation condition SssdNnh: speech from the poorerhearing side and background noise from the better-hearing side, each at an angle of $45^{\circ}$.

We used an array of 7 speakers at head level in a frontal semicircle to assess localization, as previously described by Arndt and colleagues [1,2]. The loudspeakers were set up in a range of $-90^{\circ}$ to $90^{\circ}$ with a separation angle of $30^{\circ}$. Each localization test consisted of 70 OLSA sentences presented randomly from one loudspeaker at sound levels of 59,62, 65,68 , and $71 \mathrm{~dB}$ SPL (mean sound level of $65 \mathrm{~dB}$ SPL). For each participant and each condition, the localization ability was measured as the angle error in degrees, i.e., the mean angle distance between the presentation loudspeaker and the loudspeaker identified. The angle error corresponding to the chance level performance of correct identification was $68.6^{\circ}$.

\section{Subjective assessment}

We measured the subjective outcome of CI with the German version of the standardized "Speech, Spatial and Qualities of Hearing Scale" (SSQ, version 3.1.2) [21]. SSQ evaluates speech intelligibility, spatial hearing, and quality of hearing in three sub-sections with 50 questions each (values between 0 and 10); the higher the score, the better the subjective assessment.

We evaluated the tinnitus burden with a numerical rating scale (NRS) between 0 and 10; 10 representing the highest tinnitus burden [22].

\section{Statistical analysis}

We performed a statistical analysis in $\mathrm{R}$ ( $\mathrm{R}$ Core Team 2017). We analyzed the SSD and AHL CI recipients separately. Significance was defined at a level of 0.05 .

We examined the normal distribution with the Shapiro-Wilk test. Paired $t$ tests (normal distribution) or Wilcoxon 's Ranks tests (not normal distribution) were used to compare the audiometric and subjective results between preoperative and long-term measurements (Table 1). 
Table 1 Normal distribution and statistical test

\begin{tabular}{|c|c|c|}
\hline Parameter & Normal distribution $^{1}$ & Statistical test ${ }^{2}$ \\
\hline \multicolumn{3}{|c|}{ Bone-conducted PTA4 } \\
\hline SSD & Non-parametric & Paired Wilcoxon-Ranks tests \\
\hline AHL & Non-parametric & Paired Wilcoxon-Ranks tests** \\
\hline \multicolumn{3}{|c|}{ Air-conducted PTA4 } \\
\hline SSD & Non-parametric & Paired Wilcoxon-Ranks tests \\
\hline AHL & Non-parametric & Paired Wilcoxon-Ranks tests** \\
\hline \multicolumn{3}{|c|}{ Monosyllabically speech recognition (unaided, $65 \mathrm{~dB}$ SPL) } \\
\hline SSD & Non-parametric & Paired Wilcoxon-Ranks tests \\
\hline AHL & Non-parametric & Paired Wilcoxon-Ranks tests* \\
\hline \multicolumn{3}{|c|}{ Speech recognition in noise SssdNnh } \\
\hline SSD & Parametric & Paired $t$-test*** \\
\hline AHL & Parametric & Paired $t$-test $* * *$ \\
\hline \multicolumn{3}{|c|}{ Speech recognition in noise $-\mathrm{S} 0 \mathrm{~N} 0$} \\
\hline SSD & Parametric & Paired $t$-test \\
\hline AHL & Parametric & Paired $t$-test \\
\hline \multicolumn{3}{|c|}{ Speech recognition in noise - SnhNssd } \\
\hline SSD & Parametric & Paired $t$-test \\
\hline AHL & Parametric & Paired $t$-test \\
\hline \multicolumn{3}{|c|}{ Localization of sound sources } \\
\hline SSD & Parametric & Paired $t$-test ${ }^{* * *}$ \\
\hline AHL & Non-parametric & Paired Wilcoxon-Ranks tests $* * *$ \\
\hline \multicolumn{3}{|c|}{ SSQ - Speech intelligibility } \\
\hline SSD & Parametric & Paired $t$-test*** \\
\hline AHL & Parametric & Paired $t$-test* \\
\hline \multicolumn{3}{|c|}{ SSQ - Spatial hearing } \\
\hline SSD & Parametric & Paired $t$-test $* * *$ \\
\hline AHL & Parametric & Paired $t$-test $* *$ \\
\hline \multicolumn{3}{|c|}{ SSQ - Quality of auditory impression } \\
\hline SSD & Parametric & Paired $t$-test \\
\hline AHL & Parametric & Paired $t$-test \\
\hline \multicolumn{3}{|c|}{ Tinnitus burden } \\
\hline SSD & Non-parametric & Paired Wilcoxon-Ranks tests** \\
\hline AHL & Non-parametric & Paired Wilcoxon-Ranks tests*** \\
\hline
\end{tabular}

$A H L$ asymmetric hearing loss, SSD single-sided deafness, PTA4 pure-tone average of the frequencies $500 \mathrm{~Hz}, 1 \mathrm{kHz}, 2 \mathrm{kHz}$, and $4 \mathrm{kHz}, S s s d N n h$ speech from the poorer-hearing/implanted side and noise from the better-hearing side; at an angle of $45^{\circ}$ and $-45^{\circ}$ each, SONO speech and noise from the front; at an angle of $0^{\circ}$, SnhNssd speech from the better-hearing side and noise from the poorer-hearing / implanted side; at angles of $45^{\circ}$ and $-45^{\circ}$ each, SSQ Speech, Spatial, and Qualities of Hearing Scale

${ }^{1}$ Shapiro-Wilk test; ${ }^{2}$ comparison between preoperative and long-term measurement; ${ }^{*} p<0.05 ; * * p<0.01$; $* * * p<0.001$

\section{Results}

\section{Participation and wearing behavior}

We identified $78 \mathrm{CI}$ recipient with SSD or AHL and CI experience of between six and eleven years: 41 with SSD and 37 with AHL. Figure 1 displays the participation of each identified candidate. The CI recipients questioned by telephone reported only their wearing behavior and, if applicable, causes of non-use.
Most interviewed subjects, namely 34 SSD CI recipients (34/38) and 32 AHL CI recipients (32/32), used their speech processor between 6 and $10 \mathrm{~h}$ daily (median: $8 \mathrm{~h}$ ) at six to eleven years after the initial fitting.

Four SSD CI recipients reported that they no longer wore their CI (9.8\% non-users). All non-users agreed to a telephone interview but refused further testing. The non-users reported the following causes for non-use: (1) no speech comprehension with CI, (2) fear of contamination of CI in the workplace, (3) lack of practice with the CI, and (4) 
Fig. 1 Flow chart of the participation of the CI recipients with single-sided deafness (SSD; blue) and asymmetric hearing loss (AHL; orange)
$78 \mathrm{Cl}$ recipients with single-sided deafness (SSD) and asymmetric hearing loss (AHL)

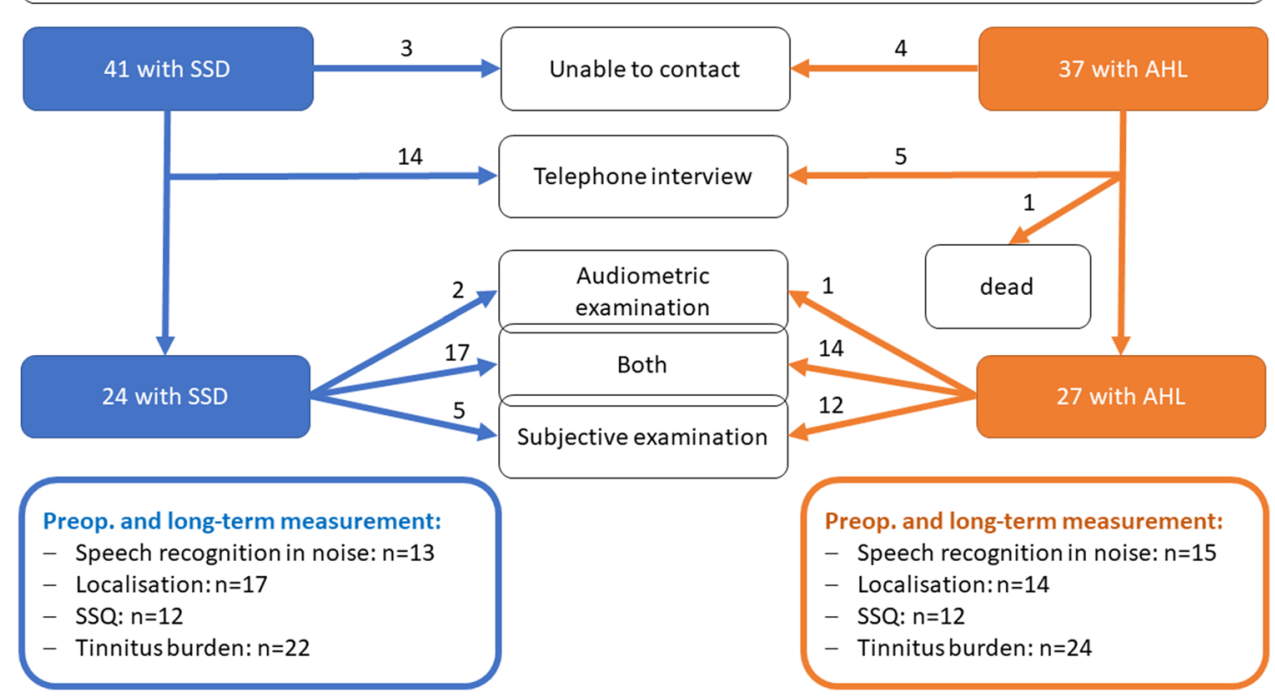

lack of subjective benefit. The non-user reporting the lack of speech comprehension was implanted with the CI one year after labyrinthitis [23]. The implantation was delayed because of the lack of coverage by the health insurance company. No AHL CI recipients reported as being non-users.

Table 2 displays the descriptive characteristics of the 24 SSD and 27 AHL CI recipients participating in either or both the audiometric and subjective examinations.

\section{Audiometric measurement}

\section{SSD CI recipients}

For the better-hearing ear, we found no difference between preoperative and long-term measurement in bone-conducted PTA4 $(6.1 \pm 4.0 \mathrm{~dB}$ SPL to $6.6 \pm 7.5 \mathrm{~dB}$ SPL), air-conducted
PTA4 $(10.9 \pm 4.9 \mathrm{~dB}$ SPL to $12.9 \pm 8.7 \mathrm{~dB}$ SPL) , and unaided monosyllabically speech recognition at $65 \mathrm{~dB}$ SPL.

In the presentation condition SssdNnh, speech recognition improved significantly from an average of $-0.6 \pm 1.9 \mathrm{~dB}$ SPL in the preoperative unaided measurement to an average of $-6.9 \pm 3.2 \mathrm{~dB}$ SPL in the long-term measurement with CI (Fig. 2a and Table 1). The presentation conditions SON0 and SnhNssd did not differ significantly (Fig. 2a).

The localization ability improved from an angle error from $33.4^{\circ}$ to $11.3^{\circ}$ (Fig. 3a and Table 1).

\section{AHL Cl recipients}

The hearing ability in the better-hearing ear decreased significantly in bone-conduction PTA4 $(21.1 \pm 8.5 \mathrm{~dB}$ SPL to $25.6 \pm 10.7 \mathrm{~dB}$ SPL), air-conduction PTA4 (26.6 $\pm 7.8 \mathrm{~dB}$

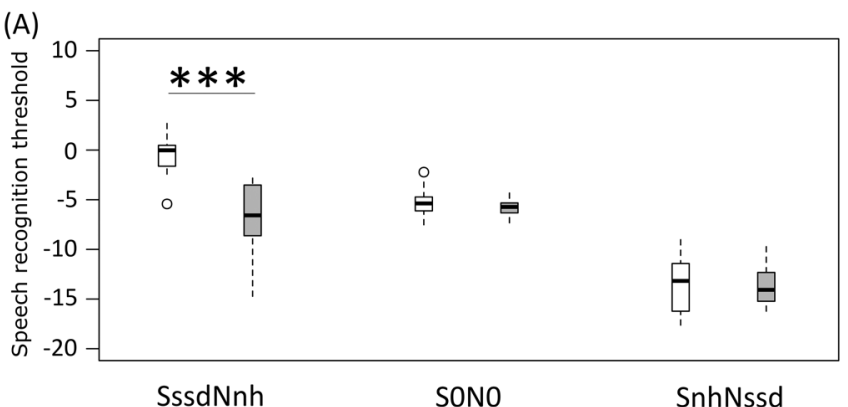

Fig. 2 Box-whisker plots of the 50\% speech recognition threshold against noise preoperatively (white) and long-term measurement after six to eleven years of CI experience (gray). SssdNnh= speech from the poorer-hearing/implanted side and noise from the better-hearing



side; at an angle of $45^{\circ}$; $\mathrm{S} 0 \mathrm{~N} 0=$ speech and noise from the front; at an angle of $0^{\circ}$; SnhNssd $=$ speech from the better-hearing side and noise from the poorer-hearing / implanted side; at angles of $45^{\circ}$. a $18 \mathrm{SSD}$ CI recipients and b 15 AHL CI recipients 
SPL to $31.9 \pm 12.1 \mathrm{~dB}$ SPL), and unaided monosyllabic speech recognition at $65 \mathrm{~dB}$ SPL (Table 1).

In the presentation condition SssdNnh, speech recognition improved significantly from an average of $0.9 \pm 3.6 \mathrm{~dB}$ SPL in the preoperative measurement to an average of $6.0 \pm 4.0 \mathrm{~dB}$ SPL in the long-term measurement (Fig. $2 \mathrm{~b}$ and Table 1). Speech recognition did not change significantly in the presentation conditions SONO and SnhNssd (Fig. 2b).

The ability to localize sounds improved significantly from an angle error of $38.1-16.7^{\circ}$ (Fig. 3b and Table 1).

\section{Subjective assessment}

\section{SSD Cl recipients}

Subjective speech intelligibility and spatial hearing in the SSQ improved significantly in the long-term measurement (Fig. 4a and Table 1). The quality of the auditory signal did not reach significance $(p=0.059)$. Of the 22 SSD CI recipients, 21 reported a preoperative tinnitus. The CI reduced


Fig. 3 Box-whisker plots of the localization of sound sources preoperatively (white) and the long-term measurement after six to eleven years of CI experience (gray). a 17 SSD CI recipients and b 14 AHL CI recipients

(A)

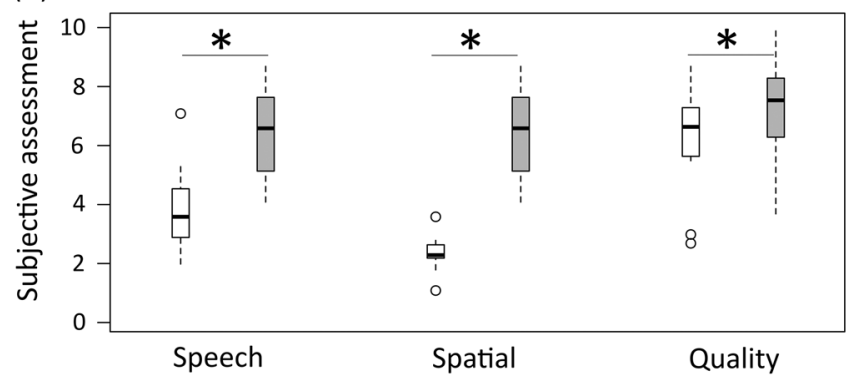

(B)



Fig. 4 Box-whisker plots of the subjective assessment on the "Speech, Spatial, and Qualities of Hearing Scale" (SSQ) preoperatively (white) and long-term measurement after six to eleven years of CI experience (gray). a 12 SSD CI recipients and b 12 AHL CI recipients

(A)



(B)

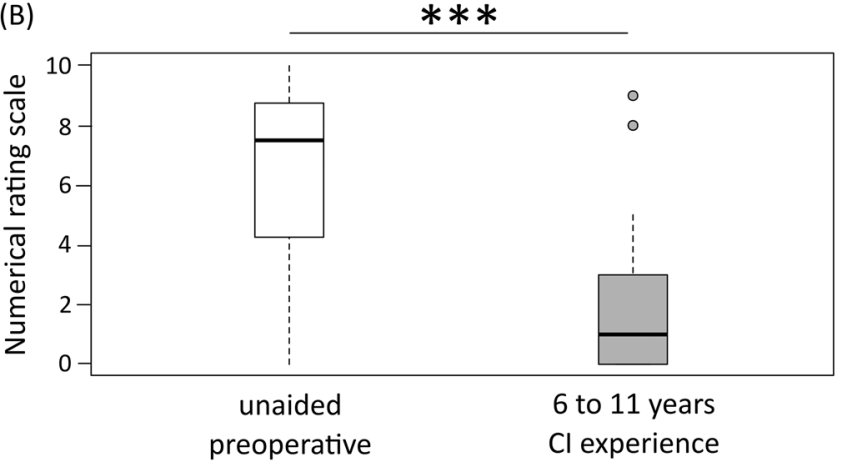

Fig. 5 Box-whisker plots of the burden of tinnitus preoperatively (white) and long-term measurement after six to eleven years of CI experience (gray). a 22 SSD CI recipients and b 24 AHL CI recipients 
the tinnitus burden significantly from $6.5 \pm 2.8$ to $3.4 \pm 2.9$

(Fig. 5a and Table 1).

\section{AHL Cl recipients}

Subjective speech comprehension and spatial hearing improved significantly in the long-term measurement (Fig. 4b and Table 1). Of 24 AHL CI recipients, 21 reported tinnitus preoperatively. We found that CI significantly reduced the tinnitus burden in AHL CI recipients from $6.2 \pm 2.1$ to $3.5 \pm 2.8$ (Fig. $5 b$ and Table 1 ).

\section{Discussion}

We have found that CI improves speech recognition in noise, the localization of sound sources, and subjective speech intelligibility and spatial hearing in AHL and SSC $\mathrm{CI}$ recipients with six to eleven years of CI experience. CI also reduces the tinnitus burden in both groups. Other than four non-users with SSD, all interviewed patients wore their CI daily.

\section{Wearing behavior}

Information on wearing behavior was available from 38 (92.7\%) of the 41 long-term SSD CI recipients and from
$32(89.2 \%)$ of the 37 AHL CI recipients. Most of the interviewed SSD (89.5\%) and all AHL (100\%) CI recipients wore their CI for six to ten hours daily. The percentage of nonusers was comparable with that for bilateral deaf patients at a rate of $2.8-13 \%[24,25]$. None of the four non-users terminated CI use because of equipment failure or perioperative complications. Three non-users reported that insufficient practice time or special workplace situations led to incomplete hearing rehabilitation and the lack of subjective benefit. We, therefore, recommend intensive preoperative education and consideration of each patient's history before cochlea implantation to prevent non-use.

\section{Audiometric measurement}

The CI enables a subject with AHL or SSD to overcome the head shadow when speech is presented on the side of the CI (SssdNnh) and thus ensures responsiveness on the CIsupplied side, even against noise. In this long-term study, we have confirmed the improvement in speech recognition against background noise in the condition SssdNnh as shown previously for shorter follow-up periods $[1,2,12,15,26$, 27].

When presenting speech and noise from the front (SONO), we determined no change in speech recognition with CI, as earlier reported by our research group after a shorter CI follow-up $[1,2]$. However, other authors have described
Table 2 Descriptive characteristics of the study groups, mean $\pm \mathrm{SD}$ or $N(\%)$

\begin{tabular}{lll}
\hline Characteristics & SSD CI recipients & AHL CI recipients \\
\hline$N$ & 24 & 27 \\
Femal & $15(63 \%)$ & $15(56 \%)$ \\
Age (years) & $50.9 \pm 10.9$ & $63.8 \pm 9.5$ \\
Duration of hearing impairment (years) & $3.5 \pm 7.4$ & $7.8 \pm 1.3$ \\
CI experience (years) & $8.7 \pm 1.9$ & $4.3 \pm 3.7$ \\
Cause of deafness & & \\
Sudden deafness & 10 & 9 \\
Labyrinthitis & 4 & 2 \\
Endolymphatic hydrops & 2 & 1 \\
Mumps infection & 1 & 1 \\
Otosclerosis & 2 & 1 \\
After ear surgery & 2 & 2 \\
Petrous bone fracture & 1 & 0 \\
Cogan syndrome & 1 & 0 \\
Measles infection & 1 & 1 \\
After acoustic neuroma resection & 0 & 1 \\
After cerebral apoplexy & 0 & 1 \\
Unknown & 0 & 8 \\
CI manufacturer & 21 & 21 \\
Cochlear & 3 & 5 \\
Med-El & 0 & 1 \\
Advanced Bionics & & \\
\hline
\end{tabular}

$A H L$ asymmetric hearing loss, $C I$ cochlear implant, $S S D$ single-sided deafness 
significant improvement in this presentation condition, even in short-term evaluations [3, 14, 15, 27]; Távora-Vieira and colleagues (2019) have also reported this effect in their longterm study. Individual differences of the patients might cause this discrepancy: subjects included in the present study also participated in the shorter follow-up studies of our research group $[1,2]$. Another contributing factor might be the conditions that were compared: Távora-Vieira and colleagues [14] compared the conditions CI "on" and "off", whereas we compared preoperative with long-term results.

Speech recognition remained stable, even when the speech was presented from the $\mathrm{NH}$ side and noise came from the CI side (SnhNssd). In this presentation condition, we expected no improvement because of the asymmetric hearing situation and former study results $[1,2,12,14,15,26]$.

$\mathrm{CI}$ improved sound localization compared with the preoperative unaided situation. This agrees with the literature describing patients with shorter [1, 2, 26-29] and with longer CI experience [14].

\section{Subjective assessment}

Speech comprehension and spatial hearing in the SSQ improved with six to eleven years of CI experience. Rating of long-term hearing quality was unchanged, similar to patients with six to twelve months of CI experience [1,2]. However, in SSD CI recipients, we saw a trend towards better long-term hearing quality, despite the different acoustic and "electrical" hearing impressions; this did not reach statistical significance. Távora-Vieira and colleagues [14, 15] demonstrated a subjective improvement in the overall score of the SSQ; the SSQ subcategories were not evaluated separately.

CI permanently reduced tinnitus for most AHL (18/21) and SSD CI recipients (15/21). In 2 AHL and five SSD CI recipients, the intensity of the tinnitus remained unchanged, and only in one AHL and one SSD CI recipient was the tinnitus burden increased after CI surgery.

\section{Strength and limitations}

The presented study has the longest follow-up period (6-11 years) of SSD CI recipients in the literature, followed by Távora-Vieira et al. (4-10 years) [14]. We are the first to compare preoperative to long-term measurements. We have been able to gain information about the wearing behavior of $92.7 \%$ of the SSD and $86.5 \%$ of the AHL CI recipients (Fig. 1).

From a total of 78 subjects, only 24 SSD and 27 AHL $\mathrm{CI}$ recipients participated in the audiological and subjective measurements. All known non-users only agreed to the telephone interview. Thus, perhaps, only the better performers agreed to audiological and subjective assessments; this bias cannot be ruled out with certainty. Because of incomplete follow-up data, e.g., 12 months after CI fitting, we were unable to include these measurements in the present study. We report wearing behavior in only two categories: "user" and "non-user". Datalogging provides more detailed information on daily device use and environments; these data were not available for most participants. In a previous study, we examined the datalogging in patients using the Nucleus 6 device. Across age groups, SSD subjects had an active CI-time of $8.07 \mathrm{~h} /$ day; the age group 18 to 65 years used their CI for $7.73 \mathrm{~h} /$ day and subjects over the age of 65 years used their CI for $10.71 \mathrm{~h} /$ day [30]. In the present study, we focused on measurements recommended by the consensus papers on the successful treatment of SSD patients [17, 18].

\section{Implications for future research}

Future studies should measure speech recognition in everyday-life relevant acoustic environments with interfering sound, e.g., in a restaurant or a busy street environment, to enhance applicability. Similarly, the localization set-up could be altered to mimic everyday life more closely. The inclusion and comparison of additional time points, e.g., 6 months and 12 months after initial fitting, would allow for the investigation of time-dependent changes in SSD and AHL CI recipients. Including further questionnaires on tinnitus, vertigo, and hearing-related quality of life might provide additional information.

\section{Conclusion}

The cochlear implant is a successful long-term treatment for subjects with SSD and AHL. Only a small number of subjects with SSD are non-users after more than 5 years. Most of them stopped using the CI within the first year after implantation. Subjects with AHL and SSD can use their CI to overcome the head shadow effect without being disturbed by noise on the CI side, even in the presence of background noise. Cochlear implants improve subjective speech intelligibility and spatial hearing while reducing the tinnitus burden.

Acknowledgements The authors thank the association "Taube Kinder lernen Hören e.V." for its strong support of the cochlear implant rehabilitation center in Freiburg. In addition, we thank the Berta-OttensteinProgramme for Clinician Scientists, Faculty of Medicine, University of Freiburg for funding our scientific work. Furthermore, we like to thank Ms. Theresa Jones for providing language assistance.

Source of funding Open Access funding enabled and organized by Projekt DEAL. We received no direct funding for the present study. Speck: Cochlear: travel reimbursement. Berta-Ottenstein-Programme for Clinician Scientists: financial support for research time. T. Wesarg: Advanced Bionics: financial support for research, travel reimbursement; Cochlear: financial support for research, travel reimbursement; 
MED-EL: financial support for research, travel reimbursement; Phonak Communications: financial support for research, travel reimbursement; Oticon Medical: travel reimbursement. A. Aschendorff: Advanced Bionics: financial support for research, medical advisory board, travel reimbursement; Cochlear: financial support for research, travel reimbursement; MED-EL: financial support for research, travel reimbursement; Oticon Medical: financial support for research, travel reimbursement. F. Hassepaß: Advanced Bionics: travel reimbursement; Cochlear: financial support for research, travel reimbursement; MEDEL: travel reimbursement. S. Arndt: Advanced Bionics: travel reimbursement; Cochlear: financial support for research, travel reimbursement; MED-EL: financial support for research, travel reimbursement; Oticon Medical: travel reimbursement. This study was conducted in accordance with the guidelines of the Declaration of Helsinki (Washington, World Medical Association, 2013). The Ethics Committee of the Albert-Ludwigs-University of Freiburg (No. 3/17) approved the present study. We registered the study with the German Register of Clinical Studies (DRKS00017632).

\section{Compliance with ethical standards}

Conflicts of interest P. Challier and T.F. Jakob: no conflicts of interest.

Informed consent All participants signed informed consent statements before inclusion in the study.

Open Access This article is licensed under a Creative Commons Attribution 4.0 International License, which permits use, sharing, adaptation, distribution and reproduction in any medium or format, as long as you give appropriate credit to the original author(s) and the source, provide a link to the Creative Commons licence, and indicate if changes were made. The images or other third party material in this article are included in the article's Creative Commons licence, unless indicated otherwise in a credit line to the material. If material is not included in the article's Creative Commons licence and your intended use is not permitted by statutory regulation or exceeds the permitted use, you will need to obtain permission directly from the copyright holder. To view a copy of this licence, visit http://creativecommons.org/licenses/by/4.0/.

\section{References}

1. Arndt S, Aschendorff A, Laszig R et al (2011) Comparison of pseudobinaural hearing to real binaural hearing rehabilitation after cochlear implantation in patients with unilateral deafness and tinnitus. Otol Neurotol 32(1):39-47

2. Arndt S, Laszig R, Aschendorff A et al (2017) Cochlea-ImplantatVersorgung von Patienten mit einseitiger Taubheit oder asymmetrischem Hörverlust. HNO 65:98-108. https://doi.org/10.1007/ s00106-016-0297-5

3. Hol MKS, Kunst SJW, Snik AFM et al (2010) Bone-anchored hearing aids in patients with acquired and congenital unilateral inner ear deafness (Baha CROS): clinical evaluation of 56 cases. Ann Otol Rhinol Laryngol 119:447-454. https://doi. org/10.1177/000348941011900704

4. Feuerstein JF (1992) Monaural versus binaural hearing: Ease of listening, word recognition, and attentional effort. Ear Hear 13:80-86. https://doi.org/10.1097/00003446-199204000-00003

5. Wie OB, Pripp AH, Tvete OE (2010) Unilateral deafness in adults: effects on communication and social interaction. Ann Otol Rhinol Laryngol 119(11):772-781

6. Lucas L, Katiri R, Kitterick PT (2018) The psychological and social consequences of single-sided deafness in adulthood. Int $\mathbf{J}$
Audiol 57:21-30. https://doi.org/10.1080/14992027.2017.13984 20

7. Subramaniam K, Eikelboom RH, Eager KM, Atlas MD (2005) Unilateral profound hearing loss and the effect on quality of life after cerebellopontine angle surgery. Otolaryngol Head Neck Surg 133:339-346. https://doi.org/10.1016/j.otohns.2005.05.017

8. Alhanbali S, Dawes P, Lloyd S, Munro KJ (2017) Self-reported listening-related effort and fatigue in hearing-impaired adults. Ear Hear 38:e39-e48. https://doi.org/10.1097/AUD.0000000000 000361

9. Andersson G, Freijd A, Baguley DM, Idrizbegovic E (2009) Tinnitus distress, anxiety, depression, and hearing problems among cochlear implant patients with tinnitus. J Am Acad Audiol 20:315-319. https://doi.org/10.3766/jaaa.20.5.5

10. Kitterick PT, O'Donoghue GM, Edmondson-Jones M et al (2014) Comparison of the benefits of cochlear implantation versus contra-lateral routing of signal hearing aids in adult patients with single-sided deafness: study protocol for a prospective withinsubject longitudinal trial. BMC Ear Nose Throat Disord 14:7. https://doi.org/10.1186/1472-6815-14-7

11. Häußler SM, Knopke S, Dudka S et al (2020) Verbesserung von Tinnitusdistress, Lebensqualität und psychologischen Komorbiditäten durch Cochleaimplantation einseitig ertaubter Patienten. HNO 68:1-10. https://doi.org/10.1007/s00106-019-0705-8

12. Buechner A, Brendel M, Lesinski-Schiedat A et al (2010) Cochlear implantation in unilateral deaf subjects associated with ipsilateral tinnitus. Otol Neurotol 31:1381-1385. https://doi. org/10.1097/MAO.0b013e3181e3d353

13. Arndt S, Laszig R, Aschendorff A et al (2011) Einseitige Taubheit und Cochleaimplantat-Versorgung. Audiologische Diagnostik und Ergebnisse HNO 59:437-446. https://doi.org/10.1007/s0010 6-011-2318-8

14. Távora-Vieira D, Rajan GP, Van De Heyning P, Mertens G (2019) Evaluating the long-term hearing outcomes of cochlear implant users with single-sided deafness. Otol Neurotol 40:E575-E580. https://doi.org/10.1097/MAO.0000000000002235

15. Távora-Vieira D, Marino R, Acharya A, Rajan GP (2015) The impact of cochlear implantation on speech understanding, subjective hearing performance, and tinnitus perception in patients with unilateral severe to profound hearing loss. Otol Neurotol 36:430-436. https://doi.org/10.1097/MAO.0000000000000707

16. Häußler SM, Köpke V, Knopke S et al (2020) Multifactorial positive influence of cochlear implantation on patients with single-sided deafness. Laryngoscope 130:500-506. https://doi. org/10.1002/lary.28007

17. Vincent C, Arndt S, Firszt JB et al (2015) Identification and evaluation of cochlear implant candidates with asymmetrical hearing loss. Audiol Neurotol 20(Suppl 1):87-89

18. Van De Heyning P, Távora-Vieira D, Mertens G et al (2017) Towards a unified testing framework for single-sided deafness studies: a consensus paper. Audiol Neurotol 21:391-398. https:// doi.org/10.1159/000455058

19. Wagener K, Brand T, Kollmeier B (1999) Entwicklung und Evaluation eines Satztests in deutscher Sprache III: Evaluation des Oldenburger Satztests. Zeitschrift für Audiol 38:86-95

20. Wagener K, Kühnel V (1999) Entwicklung und Evaluation eines Satztests in deutscher Sprache I: Design des Oldenburger Satztests. Zeitschrift für Audiol 38:4-15

21. Gatehouse S, Noble I (2004) The Speech, Spatial and Qualities of Hearing Scale (SSQ). Int J Audiol 43:85-99. https://doi. org/10.1080/14992020400050014

22. I A, B L, C H, PA T, (2012) Psychometric evaluation of visual analog scale for the assessment of chronic tinnitus. Am J Audiol. https://doi.org/10.1044/1059-0889(2012/12-0010)

23. Hassepass F, Schild C, Aschendorff A et al (2013) Clinical outcome after cochlear implantation in patients with 
unilateral hearing loss due to labyrinthitis ossificans. Otol Neurotol 34:1278-1283. https://doi.org/10.1097/MAO.0b013e3182 937ad4

24. Marshall DH (2000) Non-use of cochlear implants by post-lingually deafened adults. Cochlear Implants Int 1:18-38. https:// doi.org/10.1179/cim.2000.1.1.18

25. Bhatt YM, Green KMJ, Mawman DJ et al (2005) Device nonuse among adult cochlear implant recipients. Otol Neurotol 26:183-187

26. Firszt JB, Holden LK, Reeder RM et al (2012) Auditory abilities after cochlear implantation in adults with unilateral deafness: A pilot study. Otol Neurotol 33:1339-1346. https://doi.org/10.1097/ MAO.0b013e318268d52d

27. Hansen MR, Gantz BJ, Dunn C (2013) Outcomes after cochlear implantation for patients with single-sided deafness, including those with recalcitrant méniére's Disease. Otol Neurotol 34:16811687. https://doi.org/10.1097/MAO.0000000000000102
28. Vermeire K, Van De Heyning P (2009) Binaural hearing after cochlear implantation in subjects with unilateral sensorineural deafness and tinnitus. Audiol Neurotol 14:163-171. https://doi. org/10.1159/000171478

29. Dorman MF, Zeitler D, Cook SJ et al (2015) Interaural level difference cues determine sound source localization by singlesided deaf patients fit with a cochlear implant. Audiol Neurotol 20:183-188. https://doi.org/10.1159/000375394

30. Rauch AK, Kagermann S, Wesarg T et al (2019) Data logging evidence of cochlear implant use in single-sided and bilateral deafness. Audiol Neurotol 24:206-216. https://doi.org/10.1159/00050 2051

Publisher's Note Springer Nature remains neutral with regard to jurisdictional claims in published maps and institutional affiliations. 